\begin{tabular}{|c|c|}
\hline Title & Intracavity femtosecond-pulse compression with the addition of highly nonlinear organic materials \\
\hline Author(s) & Y amashita, Mikio; Torizuka, Kenji; Sato, Takuzo \\
\hline Citation & $\begin{array}{l}\text { Optics Letters, 13(1), } 24.26 \\
\text { https://doi.org/10.1364/OL.13.000024 }\end{array}$ \\
\hline Issue Date & 1988-01-01 \\
\hline Doc URL & http://hdl.handle.net/2115/45322 \\
\hline Rights & (c) 1988 Optical Society of A merica \\
\hline Type & article \\
\hline File Information & OL13-1_24-26.pdf \\
\hline
\end{tabular}

Instructions for use 


\title{
Intracavity femtosecond-pulse compression with the addition of highly nonlinear organic materials
}

\author{
Mikio Yamashita, Kenji Torizuka, and Takuzo Sato \\ Laser Research Section, Radio- \& Opto-Electronics Division, Electrotechnical Laboratory, Tsukuba, Ibaraki, Japan 305
}

Received February 17, 1987; accepted October 18, 1987

\begin{abstract}
It is experimentally shown that using organic materials of high nonlinear refractive index dissolved in a saturableabsorber solution permits femtosecond-pulse compression in a simple colliding-pulse mode-locked laser employing cavity-mirror dispersion adjustment.
\end{abstract}

Fast self-phase modulation 1,2 (FSPM) arising from the nonlinear refractive index (NRI) of a solvent of a saturable absorber, ethylene glycol (EG), restricts direct generation of pulses shorter than 100 fsec from a colliding-pulse mode-locked (CPM) $\mathrm{cw}$ dye laser. ${ }^{3-6}$ For shorter-pulse generation, therefore, compensation for the upchirp caused by the FSPM is always needed, and lossless dispersive elements are used. Furthermore, in order to compress pulses external to the laser, a dispersive NRI medium such as an optical fiber is used in conjunction with dispersive elements such as a grating pair. ${ }^{7,8}$ The NRI medium broadens the pulse spectrum without disturbing relative phases between modes, and the dispersive elements remove the chirp to generate transform-limited pulses.

Recently Martinez et al. ${ }^{9}$ theoretically showed that short, stable, solitonlike pulses are generated by a CPM laser operating in the balance between the FSPM that is due to EG and cavity dispersion. They further concluded that the dependence of the pulse duration on its dispersion is remarkably asymmetric. More recently, Haus and Silberberg ${ }^{10}$ pointed out that a NRI medium introduced into a CPM laser with adjustable cavity dispersion leads to shorter, though non-soliton-like, pulses. In this case, the dependence of the pulse duration on the dispersion is symmetric. However, to the authors' knowledge, no one has experimentally investigated how the introduction of a NRI medium into a practical CPM laser influences the generation of femtosecond pulses. This may be because the additional insertion of the usual NRI medium, such as an optical fiber or a Selfoc lens,${ }^{10}$ gives rise to high optical losses, dispersion broadening, and further complication of the cavity configuration. In this Letter we demonstrate that the addition of high-NRI organic materials to a solution of the saturable absorber permits intracavity pulse compression in a simple CPM laser employing cavity-mirror dispersion adjustment.

The NRI organic materials used have the following properties: (1) a higher NRI than that of the EG solution $\left.\left(n_{2}=3.0 \times 10^{-16} \mathrm{~cm}^{2} / \mathrm{W}\right)\right)^{4,9}(2)$ an ultrafast time-dependent index change by electronic hyperpolarizability, (3) high solubility for EG or a mixture of
EG and benzylalcohol, (4) no absorption around the lasing wavelength region, and (5) good photochemical stability in the solution. Recent studies of high-NRI organic materials enabled us to find suitable materials that satisfy these properties. ${ }^{11}$ The third-order molecular hyperpolarizabilities, $\gamma$, of paradimethylamino- $\beta$ nitrostyrene ${ }^{12}$ (DMA-NS) and $2^{\prime}-4^{\prime}$-methyl nitroaniline ${ }^{11}$ (MNA) are much larger than that of usual solvents (by a factor of $\sim 10^{4}$ ). $4,9,13$ Therefore, from the NRI equation, ${ }^{14} n_{2}=3 \pi N \dot{\gamma}\left(n^{2}+2\right)^{4} / 81$, where $n$ is the linear refractive index of the solvent and $N$ is the number of molecules per unit volume, we find that $n_{2}$ for both the DMA-NS solution and the MNA solution (at concentrations of $\sim 10^{-2} \mathrm{M} /$ liter) is larger than that of EG. This is true even though $N$ for both DMA-NS and MNA is smaller than that for EG (the ratio of the former to the latter is $10^{-2}-10^{-3}$ ). In this experiment, we employed a $1.1 \times 10^{-2} \mathrm{M} /$ liter solution of DMA-NS $\left(n_{2}=5.3 \times 10^{-15} \mathrm{~cm}^{2} / \mathrm{W}\right)$ and a $3.8 \times 10^{-2}$ M/liter solution of MNA $\left(n_{2}=3.0 \times 10^{-15} \mathrm{~cm}^{2} / \mathrm{W}\right)$. The NRI's for both the DMA-NS and the MNA solutions are larger than that for EG by a factor of 20-10. For good solubility, the NRI dyes were dissolved in a DODCI (in EG) solution with the help of the addition of benzylalcohol. The absorption peaks of the DMANS and MNA solutions are 475 and $410 \mathrm{~nm}$, respectively.

The simple CPM (R6G + DODCI) laser and background-free second-harmonic generation autocorrelator used are identical to those that we previously described.5,15 The intracavity dispersion was adjusted by changing the incident angle of the multilayer dielectric mirrors $\mathrm{M}_{6}$ and $\mathrm{M}_{7}$ (Fig. 1). $\mathrm{M}_{6}$ was an angledependent dispersive mirror, while $\mathbf{M}_{7}$ was an angleindependent and negligibly small dispersive mirror. These mirrors were moved simultaneously in opposite directions to maintain a constant cavity length. The dispersion of the other mirrors except that for $\mathrm{M}_{1}$ was negligibly small. $\mathrm{M}_{1}$ provides a constant dispersion. In order effectively to produce the FSPM due to the added NRI material, a straight-stream jet nozzle (thickness $\sim 40 \mu \mathrm{m}$ ) was used to create a small thick film of the DODCI and DMA-NS mixture.

Measurement of the pulse duration and of the spec- 


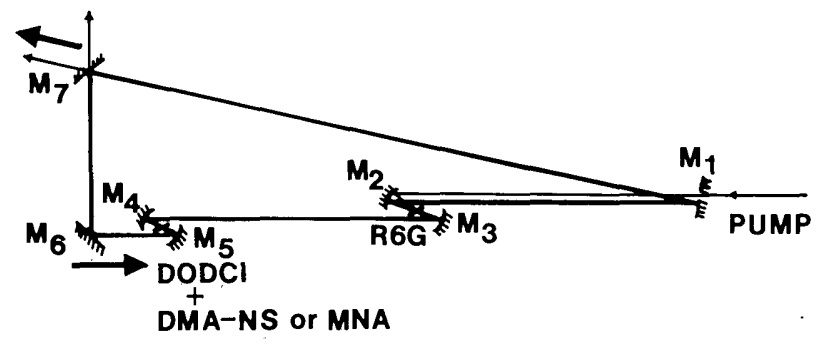

Fig. 1. Cavity configuration of the simple CPM laser. The two arrows represent the directions of the simultaneous movement of mirrors $\mathrm{M}_{6}$ and $\mathrm{M}_{7}$ for dispersion adjustment.

trum was initially carried out for the CPM laser with the $7 \times 10^{-3} \mathrm{M} /$ liter solution of DODCI absorber only. The cavity-mirror dispersion, cavity alignment, and pump power were carefully adjusted while the fastscanned autocorrelation trace and pulse spectrum were monitored on oscilloscopes. The duration of the generated pulse and the width of the relatively asymmetrical spectrum were 73 fsec and $9.1 \mathrm{~nm}$, respectively, as shown in Figs. 2(a) and 2(a'). It is assumed that instantaneous pulse profiles have a sech ${ }^{2}$ dependence. Next, the $1.1 \times 10^{-2} \mathrm{M} /$ liter DMA-NS was dissolved in the DODCI solution. To get this high-concentration solution, we first dissolved the DMA-NS in benzyl alcohol, using a magnetic stirrer. This solution was then mixed with a small amount of DODCI (in EG) solution and stirred for several hours. Finally, the solution was added to the main DODCI solution in the reservoir of a jet circulator that contained a $1-\mu \mathrm{m}$ filter. ${ }^{5}$ The high concentration did not cause the jet to clog. On addition of the DMA-NS solution, the pulse duration was reduced to 59 fsec. After adjustment of the cavity-mirror dispersion, the pulse duration was further shortened to $50 \mathrm{fsec}$, and the spectral width was broadened to $14.1 \mathrm{~nm}$. The resulting shift to shorter wavelength is shown in Fig. 2(b').

The wavelength sweep, $\Delta \lambda$, arising from the FSPM due to the $1.1 \times 10^{-2} \mathrm{M} /$ liter DMA-NS solution is approximated by $\Delta \lambda \simeq 17 n_{2} l E_{0} / \omega \tau^{2}$, where $\tau$ is the pulse duration, $\omega$ is the central angular frequency, $E_{0}$ is the intracavity pulse energy per unit area, and $l$ is the DMA-NS + DODCI film thickness. The instantaneous pulse profile is assumed to be Gaussian. The calculated value of $\Delta \lambda=10.0 \mathrm{~nm}$ suggests that the observed spectral broadening from 9.1 to $14.1 \mathrm{~nm}$ by the addition of DMA-NS is reasonable.

The dispersion dependence of the pulse duration generated from the CPM laser with the addition of the $1.1 \times 10^{-2} \mathrm{M} /$ liter solution of DMA-NS is shown in Fig. 3. It is found that with the increase of the secondorder phase dispersion, $\ddot{\phi}(\omega) \equiv \partial \phi(\omega)^{2} / \partial \omega^{2}$, the pulse duration decreases slowly, reaches a minimum, and then rapidly increases for $\phi(\omega) \gtrsim 3 \times 10^{-28} \mathrm{sec}^{2}$. The value of $\ddot{\phi}(\omega)$ for which the pulse duration is minimum is larger for the case considered here than when DMANS is not added (a case previously reported by us ${ }^{5,15}$ ), which is in agreement with theoretically derived results. ${ }^{9,10}$ The rapid increase in the pulse width for $\ddot{\phi}(\omega) \gtrsim 3 \times 10^{-28} \mathrm{sec}^{2}$ is caused not only by the excess second-order dispersion, $\ddot{\phi}(\omega)$, but also by the increase of the third-order phase dispersion, $\dddot{\phi}(\omega) \equiv \partial \phi(\omega)^{3} / \partial \omega^{3}$, of multilayer dielectric mirror $M_{6} \cdot{ }^{15}$ This is because the third-order dispersion of $\mathrm{M}_{6}$ rapidly increases over $\dddot{\phi}(\omega)=1 \times 10^{-41} \sec ^{3}$ when $\ddot{\phi}(\omega) \gtrsim 3 \times 10^{-28} \mathrm{sec}^{2}$. Miyagi and Nishida ${ }^{16}$ and others ${ }^{17}$ have presented analytic results for the effect of the third-order dispersion on short pulses. From the application of their results (Fig. 4 of Ref. 16) to pulses shorter than $50 \mathrm{fsec}$, we find that their pulses, reflected by cavity mirrors of $\dddot{\phi}(\omega) \gtrsim 1 \times 10^{-41} \mathrm{sec}^{3}$, begin to broaden and cause subpulses because of the enhancement of the effective value of $\ddot{\phi}(\omega)$ [in the case of $\ddot{\phi}(\omega), 95 \times$ enhancement occurs $^{18}$ ] by the multipass reflection in the cavity. Therefore, when $\dddot{\phi}(\omega)$ becomes negligibly small, the pulse duration will increase more slowly in the region
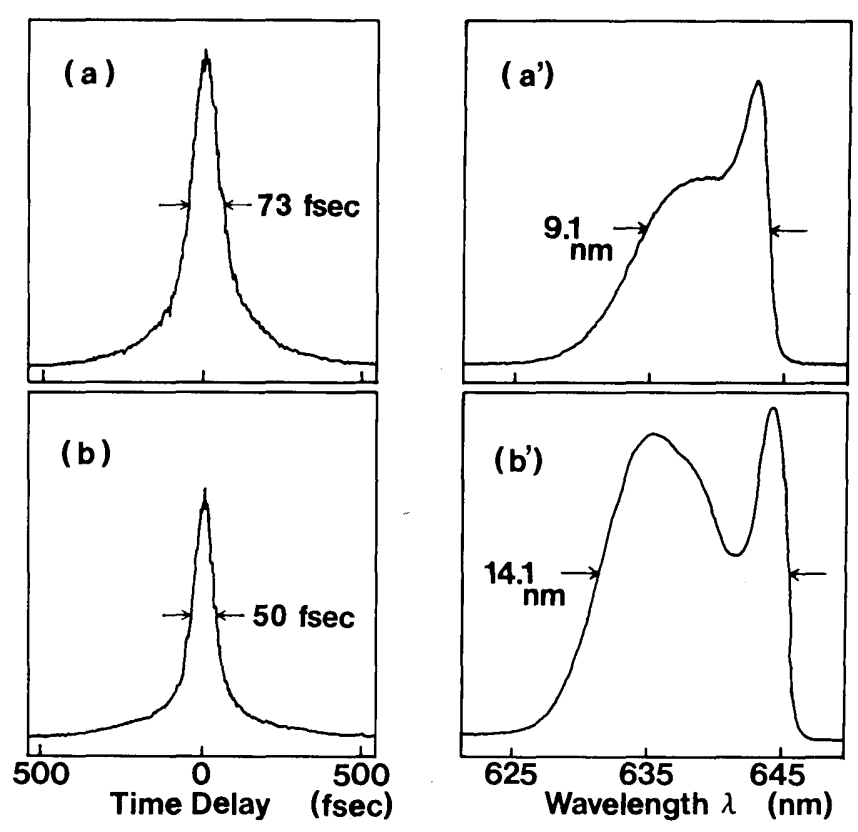

Fig. 2. Autocorrelation traces and corresponding pulse spectra from the simple CPM laser: (a), $\left(a^{\prime}\right)$ without and (b), ( $\left.b^{\prime}\right)$ with addition of $1.1 \times 10^{-2} \mathrm{M} /$ liter DMA-NS. The full width at half-maximum corresponds to a $\operatorname{sech}^{2}$ pulse duration.

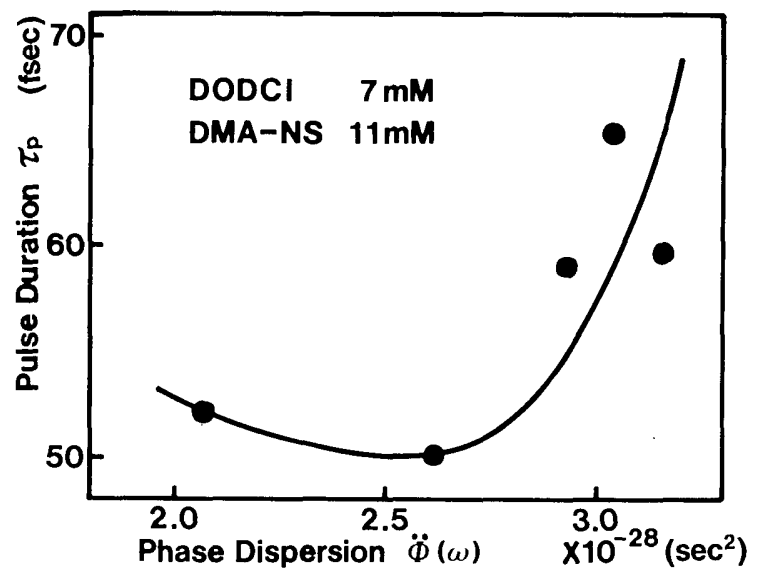

Fig. 3. Generated pulse duration as a function of cavitymirror dispersion, $\ddot{\phi}(\omega)$, in the simple CPM laser with $1.1 \times$ $10^{-2} \mathrm{M} /$ liter DMA-NS. 
$\ddot{\phi}(\omega) \gtrsim 3 \times 10^{-28} \mathrm{sec}^{2}$. This behavior is similar to the dispersion dependence of the pulse duration that was analytically derived by Haus and Silberberg ${ }^{10}$ but is unlike that discussed by Martinez et al. ${ }^{9}$ Therefore, in the present system, the pulse-shaping mechanism is not solitonlike. Consequently, pulses may be sharpened by the mechanism that was described in Ref. 10 . That is, the introduction of a chirp by the addition of the NRI material not only directly spreads the spectrum but also loosens the constraint on the time-dependent relationship between the gain, $g(t)$, and the loss, $\alpha(t)$. This is accomplished by raising the netgain curve above the loss curve. The latter effect expands the region of generation of stable pulses and hence effectively broadens the bandwidth for a given gain dispersion. Thus pulses are sharpened. In this mechanism, partial cancellation of the chirp is beneficial for the pulse duration, but completely chirp-free pulses are not necessarily the best pulses.

The same intracavity pulse-compression experiment was carried out for the CPM laser with a thinner film (flared-stream jet nozzle) of a solution of a mixture of DODCI and $3.8 \times 10^{-2} \mathrm{M} /$ liter MNA (instead of $1.1 \times 10^{-2} \mathrm{M} /$ liter DMA-NS). ${ }^{19}$ The results showed that pulses as short as 53 fsec were shortened to 48 fsec. We believe that shorter pulses are generated when this simple technique is applied to a CPM laser with elements such as prism pairs, which are adjustable for second-order dispersion but contribute little third-order dispersion.

In conclusion, it has been experimentally demonstrated for a simple CPM laser that internal femtosecond pulse compression is achieved by dissolving a high-NRI organic material in the saturable absorber and adjusting the cavity dispersion.

We are grateful to $S$. Okada for kindly supplying the DMA-NS.

\section{References}

1. R. R. Alfano and S. L. Shapiro, Phys. Rev. Lett. 24, 592 (1970).

2. R. R. Alfano and S. L. Shapiro, Phys. Rev. Lett. 24, 1217 (1970).

3. J. A. Valdmanis, R. L. Fork, and J. P. Gordon, Opt. Lett. 10, 131 (1985).

4. R. S. Miranda, G. R. Jacobovitz, C. H. B. Cruz, and M. A. F. Scarparo, Opt. Lett. 11, 224 (1986).

5. M. Yamashita, M. Ishikawa, K. Torizuka, and T. Sato, Opt. Lett. 11, 504 (1986).

6. J. Heppner and J. Kuhl, Appl. Phys. Lett. 47, 453 (1985).

7. H. Nakatsuka, D. Grishkowsky, and A. C. Balant, Phys. Rev. Lett. 47, 910 (1981).

8. W. H. Knox, R. L. Fork, M. C. Downer, R. H. Stolen, and C. V. Shank, Appl. Phys. Lett. 46, 1120 (1985).

9. O. E. Martinez, R. L. Fork, and J. P. Gordon, J. Opt. Soc. Am. B 2, 753 (1985).

10. H. A. Haus and Y. Silberberg, IEEE J. Quantum Electron. QE-22, 325 (1986).

11. For example, B. F. Levine, C. G. Bethea, C. D. Thurmond, R. L. Lynch, and J. L. Bernstein, J. Appl. Phys. 50, 2523 (1979).

12. J. L. Oudar, J. Chem. Phys. 67, 446 (1977).

13. G. R. Meredith, B. Buchalter, and C. Hanzlik, J. Chem. Phys. 78, 1543 (1983).

14. P. W. Smith, W. J. Tomlinson, D. J. Eilenberger, and P. J. Maloney, Opt. Lett. 6, 581 (1981).

15. M. Yamashita, K. Torizuka, and T. Sato, "A chirp-compensation technique using incident-angle changes of cavity mirrors in a femtosecond-pulse laser," IEEE J. Quantum Electron. (to be published).

16. M. Miyagi and S. Nishida, Appl. Opt. 18, 678 (1979).

17. D. N. Christodoulides, E. Bourkoff, and R. J. Joseph, IEEE J. Quantum Electron. QE-22, 186 (1986).

18. J. J. Fontaine, W. Dietel, and J.-C. Diels, IEEE J. Quantum Electron. QE-19, 1467 (1983).

19. M. Yamashita, K. Torizuka, T. Sato, and M. Ishikawa, in Ultrafast Phenomena V, G. R. Fleming and A. E. Siegman, eds. (Springer-Verlag, Berlin, 1986), p. 8. 